\title{
EL REGADÍO EN LAS ISLAS BALEARES USO DE AGUA DEPURADA
}

\author{
J. Enrique Santarrufina Sanmartín \\ Ministerio de Medio Ambiente y Medio Rural y Marino
}

\section{RESUMEN}

En el presente artículo se analiza la superficie regada en las Islas Baleares y la incidencia que en esta transformación ha tenido el agua depurada. Notablemente reducida con respecto a la superficie irrigada de otras Comunidades Autónomas, el regadío balear ha tenido que afrontar frecuentes sequías y ha acusado una notable falta de información, hasta que en 1995 se aprueba el Plan Nacional de Regadíos. El aumento de la superficie dedicada a cultivos mediterráneos como almendro, olivo y vid, frente al gran retroceso de forrajeras y, en general, el cambio hacia cultivos con menores requerimientos hídricos, ha reducido notablemente los consumos de agua para riego entre 1995 y 2003 de 122 $\mathrm{hm}^{3}$ a $98,09 \mathrm{hm}^{3}$, de los que 13,05 $\mathrm{hm}^{3}$ son de aguas depuradas. Al mismo tiempo, el incremento de las fórmulas de riego localizado, frente a la cada vez menor aplicación de riegos por gravedad ha contribuido, también en gran medida, a una mejor eficiencia en el uso de los caudales en las Islas Baleares. díos.

Palabras clave: Islas Baleares, regadío, aguas depuradas, Plan Nacional de Rega-

\section{ABSTRACT}

This paper analizes the irrigation area in Balearic Islands and the impact than purified waters have had in these transformations. Highly reduced from irrigated area of other regions, the balearic irrigation have fight against severe droughts and a very scarce information until the Plan Nacional de Regadios was approved. The growth in the area of Mediterranean crops, like grapes, almond and olive trees, versus the big dimminution of forage plants and, in general, the change towards less water-needed crops, have highly reduced the water irrigation consumptions between 1995 and 2003 from $122 \mathrm{hm}^{3}$ to $98^{\prime} 09 \mathrm{hm}^{3}$, from wich $13^{\prime} 05 \mathrm{hm}^{3}$ are purified waters. The increasing of localized irrigation, versus traditional one have quite contributed, to a better efficiency in water use in Balearic Islands.

Key words: Balearic Islands, irrigation, purified waters, Irrigation National Plan. 


\section{Introducción}

Es tradicional la especulación en las islas sobre la superficie regada y la demanda que conlleva. Pero ¿qué representa exactamente el regadío en las Islas Baleares? ¿Cuál es realmente la superficie regada, es decir cuál es la superficie de agricultura de regadío? ¿Cómo se riega, qué es lo que se riega, cuál es la demanda agrícola de agua y cuál el consumo agrícola? ¿Es la agricultura la responsable del posible déficit hídrico de las islas?

Históricamente, como se ha mencionado, se han venido utilizando cifras que correspondían a supuestos y extrapolaciones de datos que en gran parte eran parciales o referidos a zonas y cultivos muy concretos. Por otra parte se referían a campañas y épocas anteriores sin existir una actualización de dichas cifras ni de los datos que llevaron a ellas. Ni siquiera había una constatación con los datos oficiales del I.N.E., de tal forma que a principio de los años 90 todavía se hablaba de un consumo agrícola del $70 \%$ de los recursos totales de agua y de una superficie regada de más de 23.000 hectáreas.

De hecho nunca se había propuesto un estudio que planteara una visión completa del riego agrícola, en la que se englobaran todos los parámetros que afectan a la agricultura de regadío y a su demanda de agua: superficie, cultivos, métodos de aplicación, etc.

No fue sino hasta 1995 cuando se planteó la necesidad de realizar un estudio de caracterización del regadío para la redacción del Plan Nacional de Regadíos actualmente en vigor. En dicho estudio, el primero realizado a nivel nacional, se buscó conocer también por primera vez de una forma profunda y exhaustiva, cuánto, cómo, qué y de qué forma se riega, para así obtener los datos más fiables posibles que permitieran una planificación acorde con las circunstancias actuales. Únicamente entonces y con la mejora de los sistemas de información estadística, informática, de teledetección, etc. Se ha conseguido que, actualmente, se disponga de unos datos fiables, por no decir reales.

\section{Cuadro $\mathrm{n}^{\mathrm{o}} 1$}

EVOLUCIÓN DE LA SUPERFICIE DE REGADÍO EN LAS ISLAS BALEARES

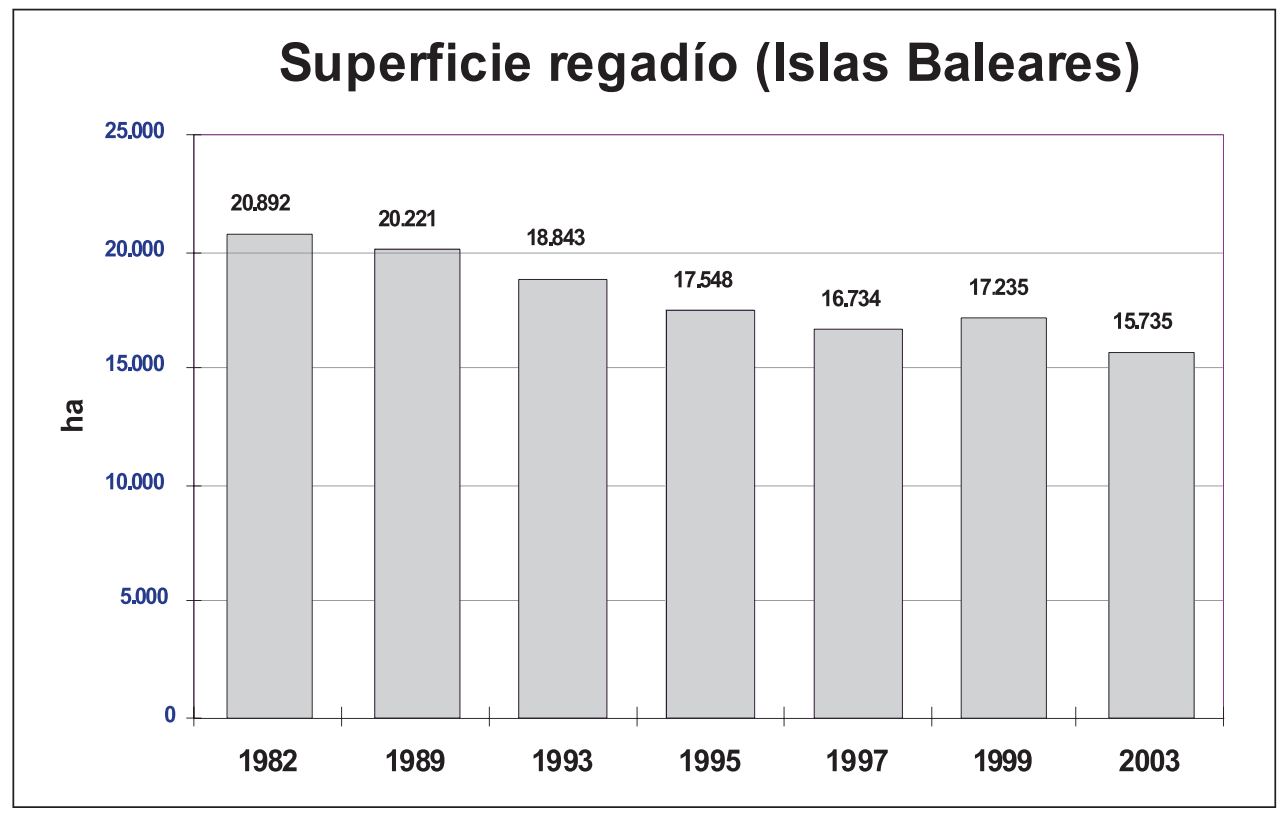


No es ningún descubrimiento destacar que las Islas Baleares sufren desde hace ya muchos años un continuo y acelerado proceso de abandono del sector agrícola siendo evidente en cuanto se refiere a la Agricultura de Regadío. Dicha circunstancia quedó clara al realizar el estudio mencionado y contrastando las cifras con los anteriores y posteriores del Censo Agrario y de las encuestas intermedias del Instituto Nacional de Estadística.

El cuadro 1 que se incluye a continuación es suficiente muestra de ello y en él se muestra claramente la tendencia decreciente de la superficie de regadío que en cifras relativas desciende un 20\% entre los años 1982-1997. La disminución absoluta desde los 80 a la actualidad se puede calcular en unas 5.000 hectáreas.

Para poder tener unos parámetros de referencia que nos sitúen sobre la importancia y la situación de la agricultura de regadío en las islas, en el cuadro 2 se exponen las superficies agrarias utilizadas y su porcentaje en regadío, existente en algunas Comunidades Autónomas.

Cuadro $\mathrm{n}^{\mathrm{o}} 2$

SUPERFICIE DE REGADÍO EN ALGUNAS COMUNIDADES AUTÓNOMAS

\begin{tabular}{|c|c|c|}
\hline \multicolumn{2}{|c|}{ SUPERFICIE DE REGADÍO EN ALGUNAS COMUNIDADES AUTÓNOMAS } \\
\hline & S A U (has) & REGABLE (\%) \\
\hline CATALUÑA & 1.138 .684 & 24 \\
\hline C. VALENCIANA & 927.000 & 41 \\
\hline C. MURCIA & 620.000 & 43 \\
\hline GALICIA & 900.045 & 15 \\
\hline CANARIAS & 142.864 & 23 \\
\hline I. BALEARS & 215.467 & 9 \\
\hline
\end{tabular}

Dejando aparte cifras de Comunidades con gran tradición en el regadío y que precisamente se encuentran a orillas del mediterráneo, es importante o significativa la comparación con el otro archipiélago, el canario, o con un territorio tan distinto climatológicamente al nuestro como es Galicia. Canarias tiene mas de 2,5 veces la superficie regable sobre Superficie Agraria útil que nosotros y Galicia más de 1,6.

Las razones se pueden resumir en dos fundamentales. Por una parte la competencia, tanto de suelo como de agua, con la demanda urbana, residencial y turística en la que indudablemente la rentabilidad del metro cúbico del agua, desde el punto de vista estrictamente monetario, es muy superior. Por otra parte se encuentra el problema del agotamiento de los acuíferos o su salinización por no citar razones de rentabilidad agrícola o de reconocimiento social. Todo ello es lo que nos ha conducido a la situación en la que nos encontramos y a la que brevemente se ha expuesto su evolución.

\section{El regadío}

Antes de iniciar un somero análisis de los datos existentes sería conveniente compartir unas reflexiones sobre el regadío tomadas en gran parte del profesor Jaime Lamo de Espinosa.

De los tres sectores económicos el industrial, el de servicios incluyendo en el todas las actividades turísticas, el agrícola y se añade el urbano, la agricultura de regadío es, efectivamente, el primer sector de demanda y consumo de agua en España. ¿Se me ocurre 
pensar si es correcto decir que la agricultura «consume»? ¿Quién consume después lo que se produce gracias a esa agua? Actualmente la agricultura demanda entre 79/80\% del agua total y consume el $70 \%$, aunque como se irá viendo, esta situación a nivel nacional, no es aplicable a esta Comunidad Autónoma. Pero este hecho, en sí, no es una excepción a nivel internacional. Lo mismo ocurre también en Francia, por ejemplo. Siendo la climatología tan diferente de la nuestra hay que destacar que dicho país ha pasado de 500.000 hectáreas regadas en el 1955 al millón y medio en 1998 y la cifra actual está muy próxima a los 1,8 millones de hectáreas si no los ha sobrepasado ya.

La grave irregularidad pluviométrica que se sufre en los países de la cuenca mediterránea, que todos conocemos y sufrimos, obliga a perseguir la mayor superficie que pueda transformarse en regadío y es lógico intentar conseguir una mayor estabilidad en la producción.

Es cierto que España es el primer país de Europa y el noveno del mundo en superficie de riego, pero esto no es, en principio, motivo de orgullo o satisfacción. Esto representa no una bendición sino una mala suerte o una desgracia. Es la desgracia de tener que luchar con el clima para poder asegurar la subsistencia, es la mala suerte de tener que trabajar denodadamente para intentar paliar los efectos de esa climatología. Entonces, cuando se consigue superar esa fase, sí que hay motivo de satisfacción al comprobar los resultados, siempre que las actuaciones realizadas lo hayan sido respetuosamente. Todo esto no viene de ahora , indudablemente, las irregularidades pluviométricas y climatológicas no son nada nuevo. A finales del siglo XIX el geólogo y paleontólogo aragonés Lucas Mallada decía «Dios nos libre de aquellas épocas en que, año tras año, las pertinaces sequías y los fríos rigurosos afligieron despiadadamente nuestra patria».

Las primeras referencias históricas del regadío, tal y como lo concebimos en la actualidad y dejando aparte las posibles pequeñas zonas o huertos de regadío anteriores, corresponden con la presencia árabe. Este es el primer momento histórico del regadío en España. Los árabes con su tradición y su cultura del agua, expresada más lúdicamente en sus inigualables jardines, marcan la expansión de la agricultura de regadío y de las técnicas imprescindibles para hacerlo posible allí donde el agua no está tan próxima. Las técnicas de los canales, acequias, aljibes, etc. coexisten con los huertos creados al amparo y en la vecindad de manantiales, pozos, galerías o torrentes. Entonces, como ahora, no era tampoco un bien ilimitado, como bien lo conocía la cultura árabe, sino un bien muy escaso. Por todo ello y debido a su importancia, con el fin de una mejor administración de los recursos se otorgan privilegios o fueros a los administradores de los mismos, antecedentes claros de las actuales Comunidades de Regantes. Como ejemplo se pueden citar los privilegios que el Rey Jaime I otorga a los acequieros de Valencia, dichos privilegios tenían el carácter de leyes y regulaban la distribución de las aguas de las acequias. Estas disposiciones, al parecer, confirmaban o actualizaban las realidades anteriores a la Reconquista.

En la isla de Mallorca también existe constancia documental de estas organizaciones, tal como se puede constatar en la historia de Soller. En la misma se incluye una sentencia del año 1293, es decir dictada durante el reinado de Jaime II, sobre un pleito existente entre los hortelanos y los molineros que eran los usuarios de las acequias del Valle, en la que, entre otras cosas se decide que: «Todas las albercas sarracenas y antiguas se hagan y construyan en argamasa y que se cuiden de tal modo que estén limpias y estancadas (que se mantengan impermeabilizadas para evitar las perdidas)». Aquí hay otro ejemplo de la herencia árabe del regadío.

¿Pero estos riegos qué finalidad tienen? Su implantación se debe, indudablemente, a la necesidad de ser productivos. De mantener a una población en crecimiento, de procurar su supervivencia. Se trata de obtener frutas y hortalizas para el consumo ordinario, en muchos de los casos el propio autoconsumo y en otros con destino al mercado más próximo. 
Actualmente se puede plantear la duda del sentido del regadío. ¿Tiene hoy el regadío la misma razón de ser que en épocas anteriores? La otra cuestión que se le plantea a no pocas personas es la conveniencia de mantener un regadío si conlleva un alto coste de medios natrurales, la pregunta consiguiente es ¿pero el coste que conlleva es tan elevado o debe serlo? ¿Defender o apoyar el regadío supone ser un nostálgico del pasado o incentivar una agricultura obsoleta?

En primer lugar había que establecer una de las relaciones más importantes EROSIÓN/DESERTIZACIÓN, el segundo el binomio agricultura/paisaje, en tercero rentabilidad/abandono, etc. Sin volver a estas relaciones ni a la realidad de que la agricultura mediterránea o es de regadío o no puede ser competitiva, se exponen algunas cifras del regadío de las Islas Baleares para que se pueda individualmente extraer las consecuencias que crean oportunas.

\section{Estado actual y evolución}

En los siguientes cuadros se exponen los últimos datos obtenidos tanto de superficies como de demanda del agua, sin entrar a considerar como demanda agrícola lo que se viene a llamar agrojardineria. Los primeros datos de referencia que se exponen fueron obtenidos dentro del estudio de caracterización del regadío citado al principio, durante la campaña de 1996 con la colaboración de la Dirección General de Recursos Hídricos y de la Conselleria de Agricultura y Pesca y contrastados posteriormente con el último Censo Agrario y las superficies registradas para las ayudas de la PAC. Los datos posteriores se han obtenido del Censo Agrario realizado en 1999 y de la Encuesta de Estructura de las Explotaciones Agrarias realizadas por el Instituto Nacional de Estadística en el año 2003.

Ese intervalo de tiempo da una tendencia clara y fiel de la evolución que sigue el regadío.

En primer lugar se estudian cuáles son los cultivos de regadío en las islas. Es decir qué es aquello que demanda agua. En el cuadro que se expone a continuación se pueden ver, divididos por grandes grupos de cultivos, las superficies ocupadas por cada uno de ellos y la evolución entre el año 1995 y 2003. En el estudio de referencia se obtuvieron datos por islas, datos de los que no disponemos para los años posteriores.

Cuadro n 3

CULTIVOS EN REGADÍO (HA)

\begin{tabular}{|c|c|c|c|c|c|c|}
\hline \multirow[t]{2}{*}{ Cultivo } & \multicolumn{4}{|c|}{1995} & \multirow{2}{*}{$\begin{array}{c}1999 \\
\text { (censo) }\end{array}$} & \multirow{2}{*}{$\begin{array}{c}2003 \\
\text { (encuesta) }\end{array}$} \\
\hline & MALLORCA & MENORCA & IBIZA & TOTAL & & \\
\hline Cereales grano & 1.461 & 25 & 75 & 1.561 & 3.205 & 3.515 \\
\hline Leguminosas grano & 105 & 10 & 29 & 144 & 165 & 156 \\
\hline Tubérculos & 1.569 & 110 & 220 & 1.899 & 1.573 & 1.937 \\
\hline Ornamentales & 121 & 0 & 2 & 123 & 154 & 68 \\
\hline Forrajeras & 5.237 & 2.128 & 431 & 7.796 & 5.394 & 2.603 \\
\hline Hortícolas & 2.998 & 181 & 395 & 3.574 & 2.653 & 2.852 \\
\hline Industriales & 61 & 0 & 1 & 62 & 230 & 255 \\
\hline Cítricos & 1.735 & 7 & 93 & 1.835 & 2.513 & 2.391 \\
\hline Frutales & 44 & 169 & 31 & 541 & 1.044 & 1.427 \\
\hline Otros & 13 & 0 & 0 & 13 & 304 & 531 \\
\hline TOTAL & 13.741 & 2.530 & 1.277 & 17.548 & 17.235 & 15.735 \\
\hline
\end{tabular}


Cuadro $\mathrm{n}^{\circ} 4$

EVOLUCIÓN DE ALGUNOS CULTIVOS

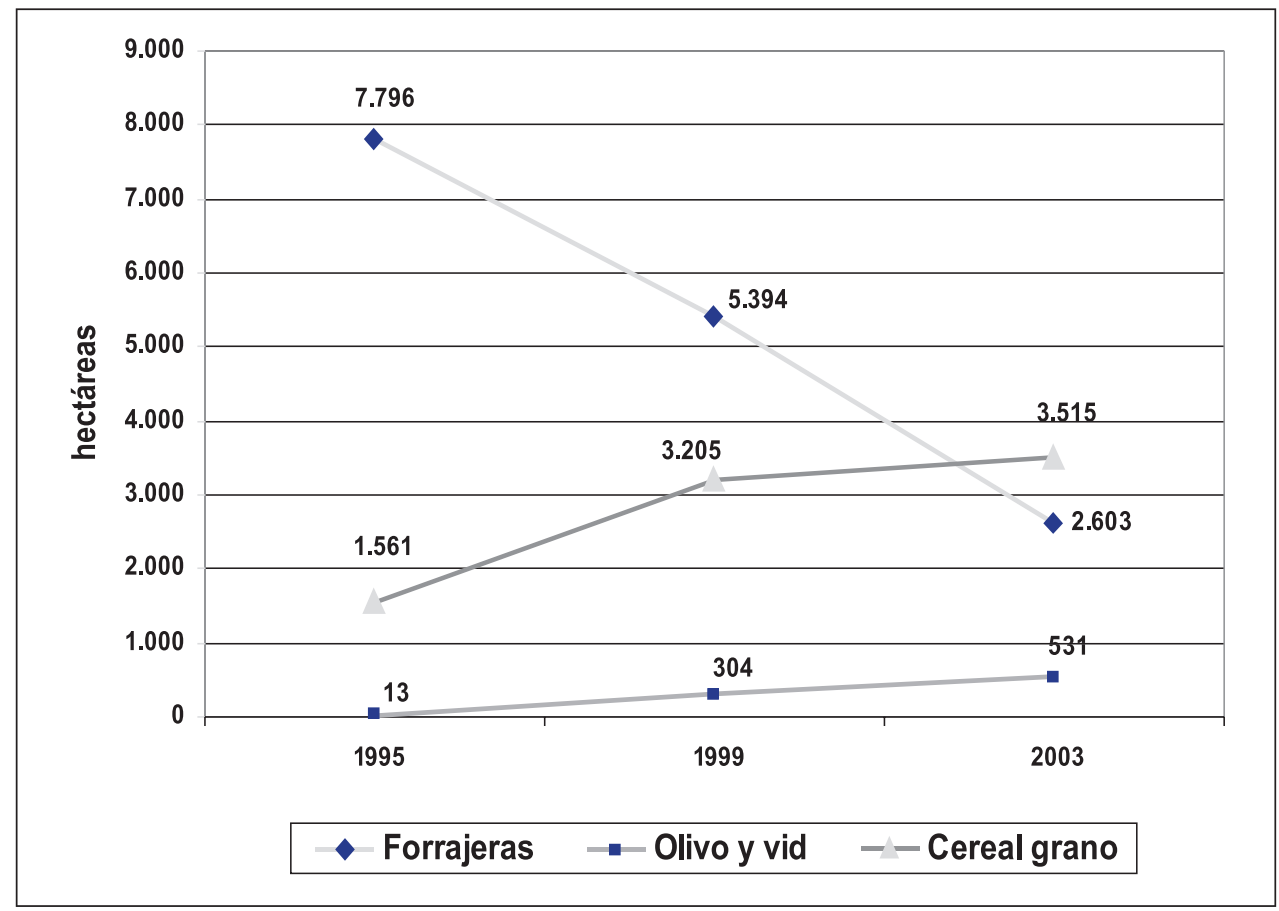

Como se puede observar al estudiar el cuadro resalta enormemente la evolución de las superficies ocupadas por algunos cultivos, así mientras que las leguminosas grano, tubérculos, hortalizas y cítricos, prácticamente no varían nos encontramos con otros en que su variación es espectacular. La superficie ocupada por el cultivo de forrajeras ha sufrido un fortísimo descenso, pues mientras en 1995 representaba el 18\% de la misma. Por el contrario los cereales en regadío han más que duplicado su superficie, pasando del $8 \%$ de la superficie regada, a representar el $22 \%$ de la misma.

La crisis del vacuno lechero y la Política Agraria Común (PAC) son indudablemente los causantes de estas variaciones.

Otro, podríamos llamar, fenómeno que hay que destacar en el incremento de la superficie regada que han protagonizado unos cultivos típicamente mediterráneos, como son el olivo, el almendro y la vid. En el año 1995 en el grupo de otros, fundamentalmente olivar y viñedo, apenas figuraban 13 hectáreas de regadío de estos cultivos y radicadas todas ellas en la Isla de Mallorca, mientras que en el año 2003 representan ya 406 hectáreas, estando el almendro dentro del grupo de frutales y causante, en parte, del aumento de superficie de regadío por 40. Es decir tenemos casi un aumento del $4000 \%$.

Conocidas las superficies que ocupan estos cultivos se puede entrar ya a estudiar la demanda de agua de los mismos y estudiar la procedencia de dicha agua y ajustar también el consumo real.

La técnica que se ha utilizado para el calculo es la siguiente, primero se han dividido las islas por áreas o zonas con los mismos tipos de datos metereológicos y características 
de suelos, aguas y cultivos. Para cada una de estas áreas se han calculado para cada cultivo sus necesidades hídricas y con todo lo anterior obtenido unas medias ponderadas para poder aplicar a las islas en general, porque evidentemente no es lo mismo la necesidad en Santa Eulalia (Ibiza), que en el Valle de Soller o en Sa Pobla que en el Pla de Sant Jordi. Por otra parte y situando el estudio en el escenario más desfavorable, se han considerado unas pérdidas del $10 \%$. Con todo lo expuesto se obtiene una demanda total y su evolución 1995-2003 como se plasma en el cuadro siguiente

Cuadro $\mathrm{n}^{\circ} 5$

CONSUMOS POR CULTIVOS EN REGADÍO $\left(\mathrm{Hm}^{3}\right)$

\begin{tabular}{|l|r|r|r|r|r|r|}
\hline \multicolumn{1}{|c|}{ CULTIVOS } & \multicolumn{4}{c|}{1995} & \multicolumn{1}{c|}{1999} & \multicolumn{1}{c|}{ 2003 } \\
\hline & MALLORCA & MENORCA & \multicolumn{1}{c|}{ IBIZA } & \multicolumn{1}{c|}{ TOTAL } & \multicolumn{1}{c|}{ TOTAL } & \multicolumn{1}{c|}{ TOTAL } \\
\hline Cereales grano & 4,54 & 0,07 & 0,16 & 4,77 & 9,83 & 10,78 \\
\hline Leguminosas grano & 0,24 & 0,01 & 0,04 & 0,29 & 0,31 & 0,29 \\
\hline Patatas & 12,38 & 0,77 & 1,82 & 14,97 & 12,58 & 15,49 \\
\hline Ornamentales & 0,46 & & 0,01 & 0,47 & 0,59 & 0,26 \\
\hline Forrajeras & 47,24 & 9,29 & 3,77 & 60,3 & 41,88 & 20,21 \\
\hline Hortícolas & 21,99 & 0,92 & 3,39 & 26,3 & 19,71 & 20,07 \\
\hline Industriales & 0,33 & 0,33 & 1,28 & 1,42 & & \\
\hline Cítricos & 11,28 & 0,02 & 0,60 & 11,90 & 16,58 & 13,96 \\
\hline Frutales & 2,71 & 0,24 & 0,15 & 3,10 & 8,09 & 13,78 \\
\hline Otros & 0,04 & 0 & 0 & 0,04 & 1,05 & 1,83 \\
\hline TOTAL & 101,21 & 11,32 & 9,94 & 122,47 & 111,90 & 98,09 \\
\hline
\end{tabular}

Es altamente significativa la evolución de la demanda de agua para riego y la causa no es únicamente por las variaciones de superficie que se han registrado en algunos cultivos, sino lo que es más importante, que lo es, por el cambio de dichos cultivos. Se han abandonado cultivos con grandes necesidades hídricas, por otros cuya demanda es mucho menor. Hay que tener en cuenta, por ejemplo, que el cereal-grano tiene un consumo de menos del $50 \%$ que la alfalfa y que los cultivos que se incorporan al riego (olivar, viñedo, almendro) demandan cantidades también muy inferiores a otros, especialmente forrajeras. 


\section{Cuadro $\mathrm{n}^{\circ} 6$ \\ CONSUMOS POR CULTIVOS EN REGADÍO}

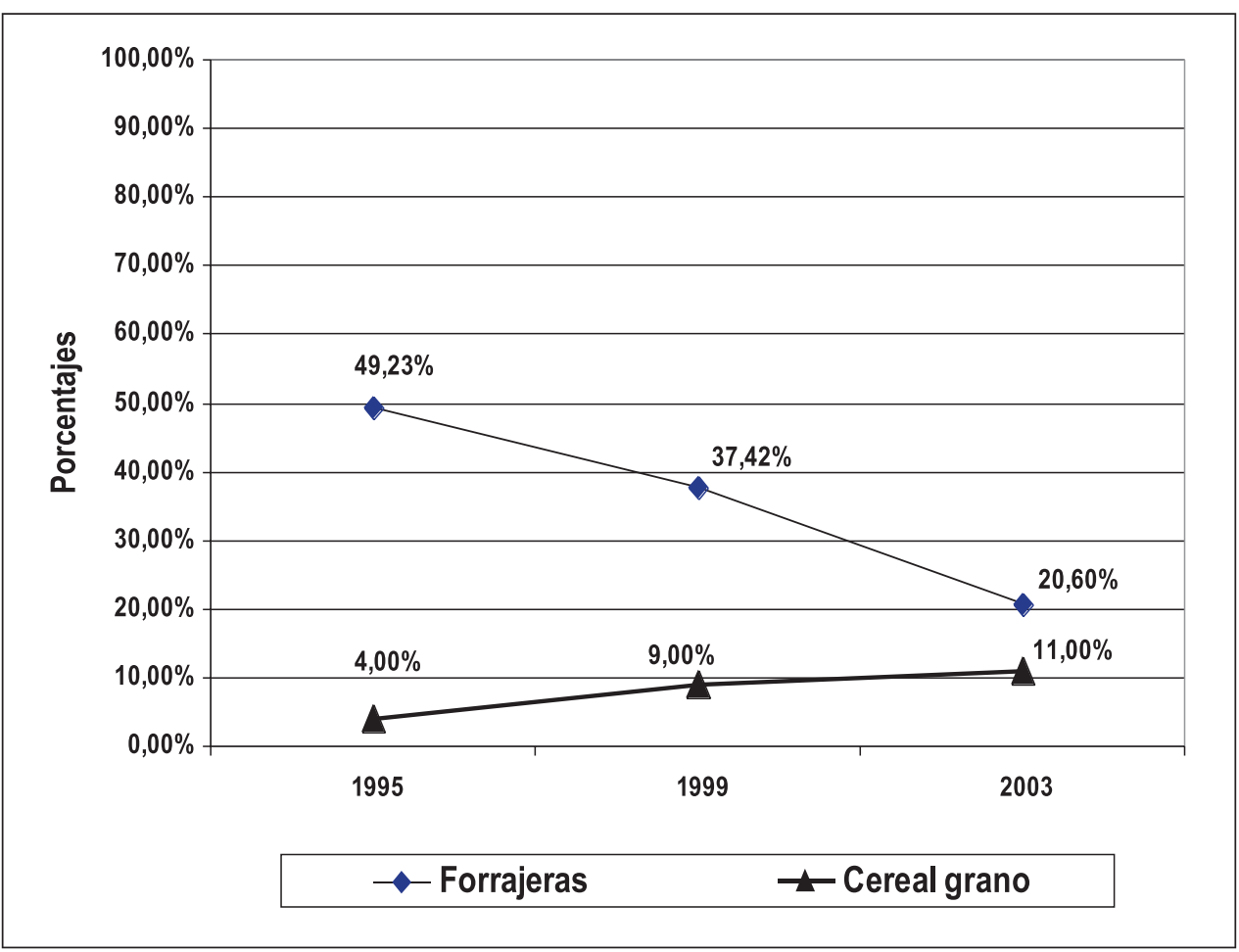

Así pues se pasa de una demanda de 122,47 $\mathrm{Hm}^{3}$ en 1995 a 98,09 $\mathrm{Hm}^{3}$, en la actualidad, lo que significa una disminución del $20 \%$.

La cuestión mas problemática, después del consumo, es indudablemente la procedencia del agua, que lógicamente en unas islas, perdón por la perogrullada, no puede ser sino de origen subterráneo salvo en el caso de la utilización de recursos no convencionales, como es éste por la reutilización del agua una vez depurada y que se tratará a continuación.

Cuadro $\mathrm{n}^{\circ} 7$

CONSUMO SEGÚN PROCEDENCIA

\begin{tabular}{|c|c|c|c|c|c|}
\hline Año & \multicolumn{2}{|c|}{ Agua Depurada } & \multicolumn{2}{c|}{ Agua Subterránea } & TOTAL \\
\hline & $\mathbf{H m}^{\mathbf{3}}$ & $\boldsymbol{\%}$ & $\mathbf{H m}^{\mathbf{3}}$ & $\mathbf{\% H m}^{\mathbf{3}}$ & \\
\hline 1995 & 13,15 & 10,73 & 109,34 & 89,27 & 122,49 \\
\hline 1999 & 13,15 & 11,86 & 97,70 & 88,14 & 110,85 \\
\hline 2003 & 13,15 & 13,40 & 84,94 & 86,60 & 98,09 \\
\hline
\end{tabular}




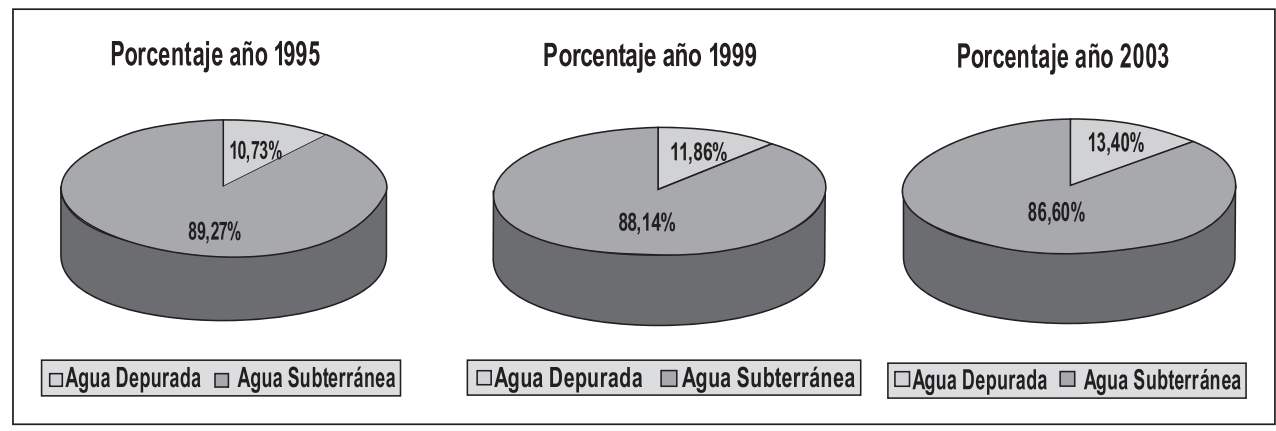

Estudiando la procedencia del agua para riego agrícola, observamos que únicamente entre $11-12 \%$ se trata de aguas residuales depuradas con un volumen total de reutilización de $13,15 \mathrm{Hm}^{3}$, que en la actualidad es un poco mayor pero que no ha tenido una variación significativa. Hay que incidir en que las cifras que se exponen se refieren a riegos agrícolas planificados, no a los que se realizan en no pocos casos de forma precaria en las cercanías de las Estaciones Depuradoras que vierten o no a torrentes. Igualmente tampoco se tratan los regadíos de campos de golf o jardines urbanos.

Por último un factor fundamental en el consumo de agua para riego es el tipo de aplicación. En las Islas Baleares la distribución que se encuentra es la siguiente:

Cuadro $\mathrm{n}^{\circ} 8$

CONSUMO SEGÚN TIPO DE APLICACIÓN

\begin{tabular}{|c|c|c|c|c|c|c|}
\hline \multirow{2}{*}{$\begin{array}{c}\text { Tipo de } \\
\text { aplicación }\end{array}$} & \multicolumn{2}{|c|}{1995} & \multicolumn{2}{c|}{1999} & \multicolumn{2}{c|}{$\mathbf{2 0 0 3}$} \\
\cline { 2 - 7 } & ha & \% & ha & \% & ha & \% \\
\hline Gravedad & 4.381 & 25,20 & 2.241 & 13,00 & 1.102 & 7,00 \\
\hline Aspersión & 9.824 & 56,51 & 9.824 & 57,00 & 8.654 & 55,00 \\
\hline Localizado & 3.180 & 18,29 & 5.170 & 30,00 & 5.979 & 38,00 \\
\hline
\end{tabular}

\begin{tabular}{|l|l|l|l|}
\hline \multicolumn{3}{|c|}{ Porcentaje año 1995} & Porcentaje año 1999 \\
\hline $18,29 \%$ & $25,20 \%$ \\
\hline $56,51 \%$ & $30,00 \%$ & $13,00 \%$ \\
\hline Gravedad $\square$ Aspersión $\square$ Localizado & $\square$ Gravedad $\square$ Aspersión $\square$ Localizado \\
\hline
\end{tabular}




\section{Consumo según tipo de aplicación}

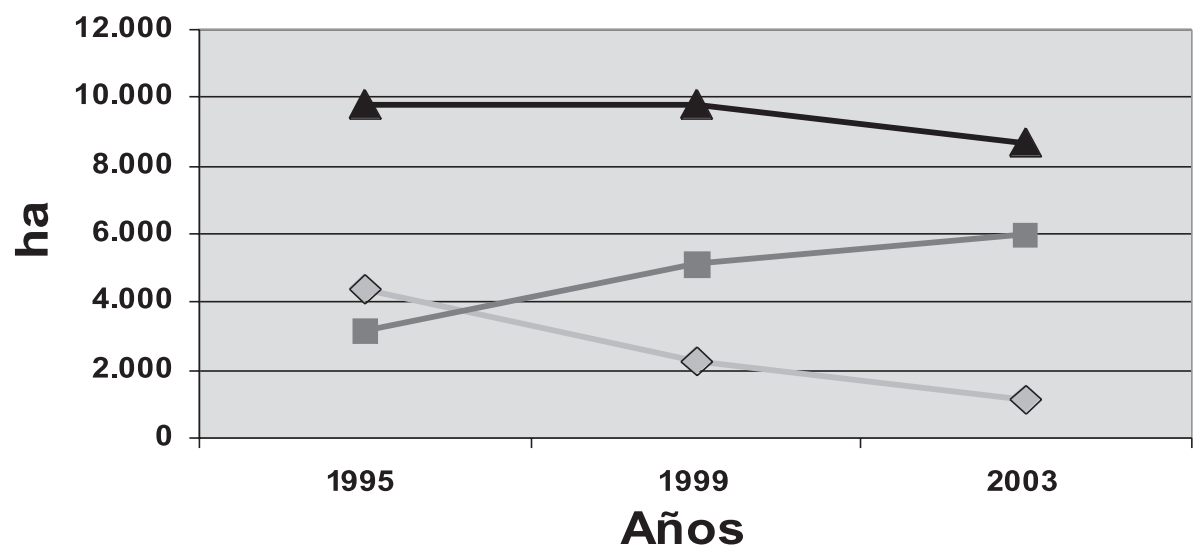

\section{Gravedad}

Se debe hacer mención, también, de la evolución de los sistemas de aplicación del riego observando como claramente se encaminan a métodos mas eficientes, con lo que es evidente el descenso del consumo.

Así mientras el riego por gravedad ha pasado de representar el $25 \%$ de la superficie a ser utilizado únicamente en el $7 \%$ de la superficie de regadío, el riego localizado (microaspersión, goteo, etc.) ha pasado de ser aplicado en el 18,29\% de la superficie a serlo en el 38\% de la misma, un incremento superior al $100 \%$.

Comparando con las medias nacionales se puede observar el extraordinario avance para el ahorro del agua en regadío que se encuentra en las islas, ya que dichas medias nos ofrecen las siguientes cifras:

Riego por gravedad - $54 \%$ de la superficie/ $7 \%$

Riego por aspersión - $19 \%$ de la superficie/ $55 \%$

Riego localizado $\quad-27 \%$ de la superficie/ $38 \%$

Contemplando cual es la demanda total en las Islas podemos calcular de una forma muy aproximada cual es el destino actual de las aguas subterraneas. Según los datos utilizados en el Plan Hidrológico de las Islas Baleares se deduce que en el año 1995 y considerando el escenario mas desfavorable, indudablemente que la situación real, se utiliza para el riego menos del $51 \%$ del agua extraída de los acuíferos.

En la actualidad la demanda para el regadío de agua subterránea y dada la evolución de la misma tal como se ha expuesto anteriormente, se encuentra en una cifra próxima al $40 \%$. 


\section{La reutilización del agua. El uso de las aguas regeneradas para el riego}

Las características hidrogeológicas de las Islas es un tema suficientemente estudiado por lo que sus circunstancias hídricas, sus posibilidades utilizando los recursos convencionales disponibles es un problema ya muy debatido y conocido, aunque se debería profundizar mucho mas sobre las actuaciones a emprender. Es pues, aquí, una insistencia inútil redundar sobre el tema.

En las islas que forman esta Comunidad Autónoma tenemos unos recursos naturales que son los que hay y no se pueden multiplicar. Tenemos los que tenemos, que son alrededor de $300 \mathrm{Hm}^{3} /$ año de los que están disponibles actualmente 260 hectómetros cúbicos, esto en periodos de pluviometría normal ya que disminuyen enormemente en los casos de sequías prolongadas. Nuestro deber y obligación es trabajar para evitar su contaminación. Desgraciadamente ya ha ocurrido en demasiados casos, que la sobreexplotación de acuíferos ha provocado la intrusión marina con la consecuente salinización de los mismos, como son las circunstancias de Campos y del Pla de Sant Jordi en Mallorca o la de la practica totalidad de la Isla de Ibiza.

Con este escenario la tendencia de la agricultura es clara: desaparición de la agricultura de regadío. Y hay que tener presente que la única agricultura que puede tener un futuro rentable en la cuenca mediterránea es la de regadío.

Nos encontramos entonces que o bien se dedican los recursos naturales de agua para actividades en las que el metro cúbico de este elemento sea más rentable, con lo cual desaparece el regadío agrícola o bien hay que disminuir la posibilidad de crecimiento tanto turístico como industrial y pone en cuestión igualmente el nuevo crecimiento vegetativo de la población, o bien el estancamiento de todos ellos. En resumen: impedir el desarrollo de las islas. Es necesario conjugar los intereses de todas las partes evitando la confrontación «Agricultura Vs. Turismo».

Nos encontramos con un desafío que creo muy atractivo: desarrollar una autentica política de gestión del agua. Esto es sencillo de decir pero debe existir una voluntad política y social que la impulse. Se trata de incorporar de forma general el agua residual regenerada a la gestión integrada de los recursos hídricos, en ciertas áreas en las que se dispone de escasos recursos como son las islas, había que exigir legalmente la reutilización, esto se encuentra ya en la normativa autónoma para los campos de golf.

La puesta en regadío de zonas lleva, lógicamente, el cultivo de las mismas evitando su erosión y desertización, no hay que decir que cuando se habla de cultivo y de regadío se habla de aplicar buenas practicas agrícolas.

Debemos, pues decantarnos por un uso planificado del agua residual regenerada porque la misma, además, comporta un avance importantísimo en la preservación de medio ambiente.

En Mallorca concretamente, la salinización de acuíferos ya provocó la primera actuación en este sentido en la década de los años setenta del siglo pasado. La creciente salinización del agua de los pozos de los agricultores de la zona impulsó como alternativa la utilización, entonces novedosa de la primera depuradora que se construyó en Palma. La primera zona regada con estas aguas fue de 250 hectáreas. Debido al éxito de la experiencia se amplio la zona con parte de la EDAR 2 de Palma hasta las 1500 hectáreas aproximadamente que son las que se riegan en la actualidad. Aquí es muy importante destacar que el efecto ha sido completamente positivo, puesto que se ha observado una clara recuperación de los niveles piezométricos y un apreciable descenso del contenido de ión cloruro en el acuífero, lo que pone de manifiesto un retroceso de la intrusión marina, según se refleja en un estudio del Instituto Geológico y Minero de España y la Junta de Aguas del año 1999. Hay que desta- 
car también que en el suelo agrícola no se ha producido ninguna alteración salvo un ligero aumento del contenido en materia orgánica, lo que por el contrario beneficia a unos suelos muy necesitados de la misma. No se han encontrado ni microorganismos potencialmente patógenos por debajo de la capa arable, ni concentraciones significativa de metales pesados, según el estudio realizado por el IRYDA en 1995.

Se debe resaltar que uno de los objetivos del Plan Hidrológico de las Islas Baleares es la reutilización al máximo de las aguas residuales una vez regeneradas, es decir aumentan los recursos no convencionales para el regadío y de forma prioritaria la sustitución de aguas subterráneas por aguas residuales depuradas (Artículo 56 de la normativa).

Para comprender la importancia que puede tener esta política hidráulica hay que tener en cuenta que la capacidad nominal de depuración de las Estaciones Depuradoras en funcionamiento es del orden de $100 \mathrm{Hm}^{3}$ y se considera que por termino medio se depuran unos $80 \mathrm{Hm}^{3} /$ año, mientras solo se reutiliza aproximadamente un $15 \%$ de los mismos. Si además tenemos en cuenta la gran inversión acometida por la Conselleria de Medio Ambiente para la ampliación y mejora de las EDAR nos encontramos en una situación optima para «multiplicar el agua». No obstante hay que destacar que si aquí solo se reutiliza ese $15 \%$ en el conjunto del estado ese porcentaje es del $0,8 \%$.

\section{El futuro}

Conscientes de lo expuesto anteriormente, en Abril de 1999 se firmó un Protocolo de inversiones para la realización de obras de regadío y optimización de recursos hídricos. Posteriormente con la aprobación y puesta en marcha del Plan Nacional de Regadíos dicho Protocolo se ha subsumido en el correspondiente Convenio para el desarrollo del Plan y se han ampliado las actuaciones a realizar. En ambos documentos se contemplan como prioritarias las obras para la reutilización de aguas.

Actualmente se encuentran ya declaradas de Interés General por parte del Ministerio de Agricultura diecisiete actuaciones, de las cuales ya han finalizado las obras de la ampliación del regadío en Santa Eulalia (Ibiza), dicho regadío tuvo una primera fase que se finalizó en la primera parte de los años noventa y ahora se han sumado unas 114 hectáreas más.

De los proyectos ya redactados y en tramitación se deduce que se incorporarán al regadío, bien por primera vez o bien por sustitución de aguas subterráneas por regeneradas unos 560 agricultores asociados en sus correspondientes Comunidades de Regantes. La superficie que representa esto es de aproximadamente 2000 hectáreas y el agua reutilizada de forma planificada ascenderá a 9,9 hectómetros cúbicos al año.

Por parte de la Conselleria de Agricultura se presentan, en principio, cuatro actuaciones directas por lo que es previsible que a la finalización del vigente convenio se llegue a alcanzar una cifra aproximada de aprovechamiento de aguas regeneradas cercana al $30 \%$. 